\title{
AN INTERACTIVE FUZZY SATISFICING METHOD \\ USING AUGMENTED MINIMAX PROBLEMS AND \\ ITS APPLICATION TO ENVIRONMENTAL SYSTEMS
}

Masatoshi Sakawa

Department of Systems Engineering

Kobe University, Japan

Hitoshi Yano

Department of Information Science

Kagawa University, Japan

RR-87-14

July 1987

Reprinted from IEEE Transactions on Systems, Man, and Cybernetics, volume 15 (1985).

INTERNATIONAL INSTITUTE FOR APPLIED SYSTEMS ANALYSIS

Laxenburg, Austria 
Research Reports, which record research conducted at IIASA, are independently reviewed before publication. However, the views and opinions they express are not necessarily those of the Institute or the National Member Organizations that support it.

Reprinted with permission from IEEE Transactions on Systems, Man, and Cybernetics 15(6):720-729. Copyright () 1985, IEEE.

All rights reserved. No part of this publication may be reproduced or transmitted in any form or by any means, electronic or mechanical, including photocopy, recording, or any information storage or retrieval system, without permission in writing from the copyright holder.

Printed by Novographic, Vienna, Austria 


\section{FOREWORD}

The International Institute for Applied Systems Analysis (IIASA) has been a leader in the area of multiobjective decisionmaking under conditions of uncertainty, and we have long been aware of Professor Sakawa's interesting work in this area. Indeed, he was invited to present a lecture here in late 1985, addressing many of the same issues raised in the appended paper.

Here, Professor Sakawa and his coauthor propose a new interactive fuzzy satisficing method for multiobjective nonlinear programming, in the case where the decisionmaker holds fuzzy goals for each of the objective functions. On the basis of this innovative method, the authors wrote a time-sharing computer program to implement man-machine interactive procedures, and this program is applied to the industrial pollution control problem, in Osaka City, Japan. Thus, the authors' ideas are in the spirit of the research that IIASA is currently carrying out on the role of analysis in the solution of real-world problems.

ALEXANDER B. KURZHANSKI Leader

System and Decision Sciences 


\title{
An Interactive Fuzzy Satisficing Method Using Augmented Minimax Problems and Its Application to Environmental Systems
}

\author{
MASATOSHI SAKAWA AND HITOSHI YANO
}

\begin{abstract}
A new interactive fuzzy satisficing method for multiobjective nonlinear programming is presented, by considering that the decisionmaker (DM) has fuzzy goals for each of the objective functions. Through the interaction with the DM, the fuzzy goals of the DM are quantified by eliciting corresponding membership functions. In order to generate a candidate for the satisficing solution (Pareto optimal) after determining the membership functions, if the DM specifies his / her reference membership values, the augmented minimax problem is solved. The DM is thus supplied with the corresponding Pareto optimal solution together with the trade-off rates between the membership functions. Then by considering the curren values of the membership functions as well as the trade-off rates, the DM acts on this solution by updating his / her reference membership values. In this way the satisficing solution for the DM can be derived efficiently from among a Pareto optimal solution set by updating his/her reference membership values. On the basis of the proposed method, a time-sharing computer program is written to implement man-machine interactive procedures. An application to the industrial pollution control problem in Osaka City in Japan is demonstrated together with the computer output.
\end{abstract}

\section{INTRODUCTION}

TN MULTIOBJECTIVE decisionmaking problems (MDMP), multiple objectives are usually noncommensurable and cannot be combined into a single objective. Moreover, the objectives usually conflict with each other in that any improvement of one objective can be achieved only at the expense of another. Consequently, the aim is to find a compromise or satisficing solution of a decisionmaker (DM), which is also Pareto optimal based on his/her subjective value judgement [1], [2], [6], [9]-[12], [14], [40]. Three most promising types of approaches for the determination of a compromise or satisficing solution of MDMP have been developed, which are (1) goal programming approaches [3], [4], [15], [16], [20]; (2) interactive approaches [8], [24]-[29], [33], [34], [36], [37], [38], [44]; and (3) fuzzy programming approaches [13], [19], [30]-[32], [41]-[43].

The goal-programming approaches, which assume that the DM can specify his/her goals of the objective functions, first appeared in 1961 text by Charnes and Cooper [3] in order to deal with multiobjective linear programming

Manuscript received December 8, 1984; revised July 7, 1985

M. Sakawa is with the Department of Systems Engineering, Faculty of Engineering, Kobe University, Kobe 657, Japan.

H. Yano is with the Department of Information Science, College of Economics, Kagawa University, Kagawa 760, Japan.
(MOLP) problems. Subsequent works on goal programming approaches have been numerous including [4], [15], [16], and [20].

The interactive approaches, which assume that the DM is able to give some preference information on a local level to a particular solution, were first initiated by Geoffrion et al. [8] and further developed by many researchers such as [24]-[29], [33], [34], [36-38], [44].

The fuzzy programming approaches, which assume that the fuzzy goals of the DM can be quantified by eliciting his/her membership functions, were first introduced by Zimmermann [41] in solving MOLP problems and further extended by several investigators such as [13], [19], [30]-[32], [42], [43].

Naturally, each of these approaches has its own advantages and disadvantages relative to the others. Therefore in this paper we present a new interactive fuzzy satisficing method by incorporating the desirable features of both the goal programming approaches and the interactive approaches into the fuzzy approaches. After determining the membership functions for each of the objective functions through the interaction with the DM, if the DM specifies his/her reference membership values, the augmented minimax problem is solved, and the DM is supplied with the corresponding Pareto optimal solution and the trade-off rates between the membership functions. Then by considering the current values of the membership functions together with the trade-off rates, the DM responds by updating his/her reference membership values and the satisficing solution for the DM can be derived efficiently from among a Pareto optimal solution set. On the basis of the proposed method, a time-sharing computer program is written in Fortran to implement man-machine interactive procedures. The industrial pollution control problem in the industrialized areas near Osaka City in Japan is formulated, and the interaction processes are demonstrated along with the computer outputs.

\section{Interactive Fuzzy Satisficing DECISIONMAKING}

In general, the multiobjective nonlinear programming (MONLP) problem is represented as the following 
vector-minimization problem:

$\min f(x) \triangleq\left(f_{1}(x), f_{2}(x), \cdots, f_{k}(x)\right)^{T}$

subject to $x \in X=\left\{x \mid x \in E^{n}, g_{j}(x) \leqslant 0, j=1, \cdots, m\right\}$

where $x$ is an $n$-dimensional vector of the decision variables $f_{1}(x), \cdots, f_{k}(x)$ are $k$ distinct objective functions of the decision vector $x, g_{1}(x), \cdots, g_{m}(x)$ are $m$ inequality constraints, and $X$ is the feasible set of constrained decisions.

Fundamental to the MONLP is the Pareto optimal concept, which is also known as a noninferior solution. Qualitatively, a Pareto optimal solution of the MONLP is one where any improvement of one objective function can be achieved only at the expense of another. Mathematically, a formal definition of a Pareto optimal solution to the MONLP is given in the following.

Definition 1 (Pareto optimal solution): A decision $x^{*} \in$ $X$ is said to be a Pareto optimal solution to the MONLP, if and only if there does not exist another $x \in X$ such that $f_{i}(x) \leqslant f_{i}\left(x^{*}\right), i=1, \cdots k$, with strict inequality holding for at least one $i$.

In practice, however, since only local solutions are guaranteed in solving a scalar optimization problem by any standard optimization technique, unless the problem is convex, we deal with local Pareto optimal solutions instead of global Pareto optimal solutions. The concept of local Pareto optimal solutions was first introduced by Geoffrion [7].

Definition 2 (local Pareto optimal solution): A decision $x^{*} \in X$ is said to be a local Pareto optimal solution to the MONLP if and only if there exists an $r>0$ such that $x^{*}$ is Pareto optimal in $X \cap N\left(x^{*}, r\right)$, i.e. there does not exist another $x \in X \cap N\left(x^{*}, r\right)$ such that $f_{i}(x) \leqslant f_{i}\left(x^{*}\right), i=$ $1, \cdots, k$, with strict inequality holding for at least one $i$, where $N\left(x^{*}, r\right)$ denotes the set $\left\{x \mid x \in E^{n},\left\|x-x^{*}\right\|<r\right\}$.

Usually, local Pareto optimal solutions consist of an infinite number of points, and some kinds of subjective judgement should be added to the quantitative analyses by the DM. The DM must select his/her local compromise or satisficing solution from among local Pareto optimal solutions.

In this paper, assuming that the DM has imprecise or fuzzy goals for each of the objective functions in the MONLP, we propose a new interactive fuzzy satisficing method.

In a minimization problem, a fuzzy goal stated by the DM may be to achieve "substantially less" than $A$. This type of statement can be quantified by eliciting a corresponding membership function.

In order to elicit a membership function $\mu_{f_{i}}(x)$ from the $\mathrm{DM}$ for each of the objective functions $f_{i}(x), i=1, \cdots, k$ we first calculate the individual minimum $f_{i}^{\min }$ and maximum $f_{i}^{\max }$ of each objective function $f_{i}(x)$ under given constraints. By taking account of the calculated individual minimum and maximum of each objective function, the DM must determine his/her subjective membership func- tion $\mu_{f_{i}}(x)$ which is a strictly monotonically decreasing function with respect to $f_{i}(x)$. Here, it is assumed that $\mu_{f_{i}}(x)=0$ or $\rightarrow 0$, if $f_{i}(x) \geqslant f_{i}^{0}$ and $\mu_{f}(x)=1$ or $\rightarrow 1$, if $f_{i}(x) \leqslant f_{i}^{1}$, where $f_{i}^{0}$ is an unacceptable level for $f_{i}(x)$, and $f_{i}^{1}$ is a totally desirable level for $f_{i}(x)$ within $f_{i}^{\text {min }}$ and $f_{i}^{\max }$.

After determining the membership functions for each of the objective functions, in order to generate a candidate for the satisficing solution, which is also local Pareto optimal, the DM is asked to specify his/her reference levels of achievement of the membership functions, called reference membership values, which can be viewed as an obvious extension of the idea of the reference point of Wierzbicki [37]. For the DM's reference membership values $\hat{\mu}_{f_{i}}, i=$ $1, \cdots k$, the corresponding local Pareto optimal solution, which is in a sense close to his/her requirement (or better, if the reference membership values are attainable), can be obtained by solving the following augmented minimax problem, where it is assumed that the difference $\left(\hat{\mu}_{f_{i}}-\right.$ $\left.\mu_{f_{i}}(x)\right)$ is of equal importance to the DM. Thus

$$
\min _{x \in X}\left\{\max _{1 \leqslant i \leqslant k}\left(\hat{\mu}_{f_{i}}-\mu_{f_{i}}(x)\right)+\rho \sum_{i=1}^{k}\left(\hat{\mu}_{f_{i}}-\mu_{f_{i}}(x)\right)\right\}
$$

or equivalently

$$
\left.\begin{array}{l}
\min _{v, x \in X} v+\rho \sum_{i=1}^{k}\left(\hat{\mu}_{f_{i}}-\mu_{f_{i}}(x)\right) \\
\text { subject to } \\
\quad \hat{\mu}_{f_{i}}-\mu_{f_{i}}(x) \leqslant v, \quad i=1, \cdots, k
\end{array}\right\}
$$

or

$$
\left.\begin{array}{l}
\min _{w, x \in X} w \\
\text { subject to } \\
\hat{\mu}_{f_{i}}-\mu_{f_{i}}(x) \leqslant w-\rho \sum_{i=1}^{k}\left(\hat{\mu}_{f_{i}}-\mu_{f_{i}}(x)\right), i=1, \cdots, k .
\end{array}\right\}
$$

The term augmented is adopted because the term

$$
\rho \sum_{i=1}^{k}\left(\hat{\mu}_{f_{i}}-\mu_{f_{i}}(x)\right)
$$

is added to the usual minimax problem, where $\rho$ is a sufficiently small positive scalar. Thus the augmented minimax problem is a natural extension of the usual minimax problem. Such an augmented minimax problem can be viewed as a modified fuzzy version of the augmented weighted Tchebycheff norm problem of Steuer and Choo [36] or Choo and Atkins [5].

It should be emphasized here that the augmented minimax problem is simply used as a means of generating a local Pareto optimal solution, and if the DM is not satisfied with the current local Pareto optimal solution, it is possible for him/her to improve the solution by updating his/her reference membership values.

The relationships between the local optimal solutions of the augmented minimax problem and the local Pareto 
optimal concept of the MONLP can be characterized by the following theorems.

Theorem 1: If $x^{*}$ is a local optimal solution to the augmented minimax problem for some $\hat{\mu}_{f}=\left(\hat{\mu}_{f_{1}}, \cdots, \hat{\mu}_{f_{k}}\right)$, then $x^{*}$ is a local Pareto optimal solution to the MONLP.

Proof: Assume that $x^{*}$ is not a local Pareto optimal solution to the MONLP, then there exists $\bar{x} \in X \cap$ $N\left(x^{*}, r\right)$ such that $f(\bar{x}) \leqslant f\left(x^{*}\right)$ or equivalently $\mu_{f}(\bar{x}) \geqslant$ $\mu_{f}\left(x^{*}\right)$ or $\hat{\mu}_{f}-\mu_{f}(\bar{x}) \leqslant \hat{\mu}_{f}-\mu_{f}\left(x^{*}\right)$, where $\mu_{f}(x)=$ $\left(\mu_{f_{1}}(x), \cdots, \mu_{f_{k}}(x)\right)$. Then it holds that

$$
\begin{gathered}
\max _{1 \leqslant i \leqslant k}\left(\hat{\mu}_{f_{i}}-\mu_{f_{i}}(\bar{x})\right) \leqslant \max _{1 \leqslant i \leqslant k}\left(\hat{\mu}_{f_{i}}-\mu_{f_{i}}\left(x^{*}\right)\right) \\
\rho \sum_{i=1}^{k}\left(\hat{\mu}_{f_{i}}-\mu_{f_{i}}(\bar{x})\right)<\rho \sum_{i=1}^{k}\left(\hat{\mu}_{f_{i}}-\mu_{f_{i}}\left(x^{*}\right)\right) .
\end{gathered}
$$

This means that

$$
\begin{aligned}
\max _{1 \leqslant i \leqslant k}\left(\hat{\mu}_{f_{i}}\right. & \left.-\mu_{f_{i}}(\bar{x})\right)+\rho \sum_{i=1}^{k}\left(\hat{\mu}_{f_{i}}-\mu_{f_{i}}(\bar{x})\right) \\
& <\max _{1 \leqslant i \leqslant k}\left(\hat{\mu}_{f_{i}}-\mu_{f_{i}}\left(x^{*}\right)\right)+\rho \sum_{i=1}^{k}\left(\hat{\mu}_{f_{i}}-\mu_{f_{i}}\left(x^{*}\right)\right)
\end{aligned}
$$

which contradicts the fact that $x^{*}$ is a local optimal solution to the augmented minimax problem (2). Hence $x^{*}$ is a local Pareto optimal solution to the MONLP.

Theorem 2: If $x^{*} \in X$ is a local Pareto optimal solution to the MONLP with $0<\mu_{f_{i}}\left(x^{*}\right)<1$ holding for all $i$, then there exists $\hat{\mu}_{f}=\left(\hat{\mu}_{f_{1}}, \cdots, \hat{\mu}_{f_{k}}\right)$ such that $x^{*}$ is a local optimal solution to the augmented minimax problem.

Proof: Assume that $x^{*}$ is not a local optimal solution to the augmented minimax problem (2) for any $\hat{\mu}_{f}$ satisfying

$$
\hat{\mu}_{f_{1}}-\mu_{f_{1}}\left(x^{*}\right)=\cdots=\hat{\mu}_{f_{k}}-\mu_{f_{k}}\left(x^{*}\right) .
$$

Then there exists $\bar{x} \in X \cap N\left(x^{*}, r\right)$ such that

$$
\begin{aligned}
\max _{1 \leqslant i \leqslant k}\left(\hat{\mu}_{f_{i}}-\mu_{f_{i}}\left(x^{*}\right)\right)+\rho \sum_{i=1}^{k}\left(\hat{\mu}_{f_{i}}-\mu_{f_{i}}\left(x^{*}\right)\right) \\
\geqslant \max _{1 \leqslant i \leqslant k}\left(\hat{\mu}_{f_{i}}-\mu_{f_{i}}(\bar{x})\right)+\rho \sum_{i=1}^{k}\left(\hat{\mu}_{f_{i}}-\mu_{f_{i}}(\bar{x})\right) .
\end{aligned}
$$

This implies that

$$
\max _{1 \leqslant i \leqslant k}\left(\mu_{f_{i}}\left(x^{*}\right)-\mu_{f_{i}}(\bar{x})\right)+\rho \sum_{i=1}^{k}\left(\mu_{f_{i}}\left(x^{*}\right)-\mu_{f_{i}}(\bar{x})\right) \leqslant 0 .
$$

Now if either any $\mu_{f}\left(x^{*}\right)-\mu_{f_{i}}(\bar{x})$ is positive or all $\mu_{f}\left(x^{*}\right)$ $-\mu_{f_{i}}(\bar{x}), i=1, \cdots, k$, are zero, this inequality would be violated for sufficiently small positive $\rho$. Hence

$$
\mu_{f_{i}}\left(x^{*}\right)-\mu_{f_{i}}(\bar{x}) \leqslant 0, \quad i=1, \cdots, k
$$

must hold. Since by the assumption $0<\mu_{f}\left(x^{*}\right)<1$, we have $f\left(x^{*}\right) \geqslant f(\bar{x})$, which contradicts the fact that $x^{*}$ is a local Pareto optimal solution to the MONLP and the theorem is proved.

As can be seen from the above proofs, it should be noted here that an obvious advantage of the augmented minimax problem over the usual minimax problem is that local Pareto optimality is guaranteed even if the uniqueness assumption for the solution is absent, because of the presence of the augmented term.

The geometric interpretation of the augmented minimax problem is as follows. Let us assume that $\left(w^{*}, x^{*}\right)$ be an optimal solution to the augmented minimax problem (4) with all the inequality constraints of (4) active. Then the intersection of the $k$ active inequality constraints in the membership function space can be obtained by solving the following simultaneous equation:

$$
\begin{gathered}
{\left[\begin{array}{cccc}
1+\rho & \rho & \cdots & \rho \\
\rho & 1+\rho & \cdots & \rho \\
\vdots & \vdots & \ddots & \vdots \\
\rho & \rho & \cdots & 1+\rho
\end{array}\right]\left[\begin{array}{c}
\mu_{f_{1}}(x) \\
\mu_{f_{2}}(x) \\
\vdots \\
\mu_{f_{k}}(x)
\end{array}\right]} \\
=\left[\begin{array}{c}
\hat{\mu}_{f_{1}}+\rho \sum_{i=1}^{k} \hat{\mu}_{f_{i}}-w^{*} \\
\hat{\mu}_{f_{2}}+\rho \sum_{i=1}^{k} \hat{\mu}_{f_{i}}-w^{*} \\
\cdots \\
\hat{\mu}_{f_{k}}+\rho \sum_{i=1}^{k} \hat{\mu}_{f_{i}}-w^{*}
\end{array}\right]
\end{gathered}
$$

Denoting the left-hand matrix by $A$, its determinant becomes $\operatorname{det}(A)=1+k \rho \neq 0$. Also, denoting the adjugate matrix of $A$ by $\left[\tilde{a}_{i j}\right]$, we have $\tilde{a}_{i i}=1+(k-1) \rho, \tilde{a}_{j i}=$ $-\rho$. Therefore the solution to the simultaneous equation (5) is represented by

$$
\mu_{f_{i}}(x)=\left(\sum_{j=1}^{k} \tilde{a}_{j i}\left(\hat{\mu}_{f_{j}}+\rho \sum_{l=1}^{k} \hat{\mu}_{f_{l}}-w^{*}\right)\right) / \operatorname{det}(A)
$$

or, using the above

$$
\mu_{f_{i}}(x)=\hat{\mu}_{f_{i}}-w^{*} /(1+k \rho) .
$$

Added insight can be obtained by comparing the isoquants of the minimax problem and the augmented minimax problem as depicted in Fig. 1, where, in general

$$
\theta=\tan ^{-1}(\sqrt{k-1} \rho /(1+\rho)) .
$$

This relation shows that $\theta$ is monotone increasing with respect to $\rho$. Thus for sufficiently small positive scalar, augmented minimax problems overcome the possibility to generate weak Pareto optimal solutions as was shown in Theorems 1 and 2. Hence augmented minimax problems are attractive for generating Pareto optimal solutions even if appropriate convexity assumptions are absent.

To verify the relation (7), we merely observe that the cosine of the angle $\theta$ between the normal vector $(-\rho, \cdots,-\rho,-1-\rho,-\rho, \cdots,-\rho)$ of the isoquant of the augmented minimax problem and the normal vector $(0, \cdots, 0,-1,0, \cdots, 0)$ of the isoquant of the minimax problem is given by $\cos \theta=(1+\rho) / \sqrt{1+2 \rho+k \rho^{2}}$. 


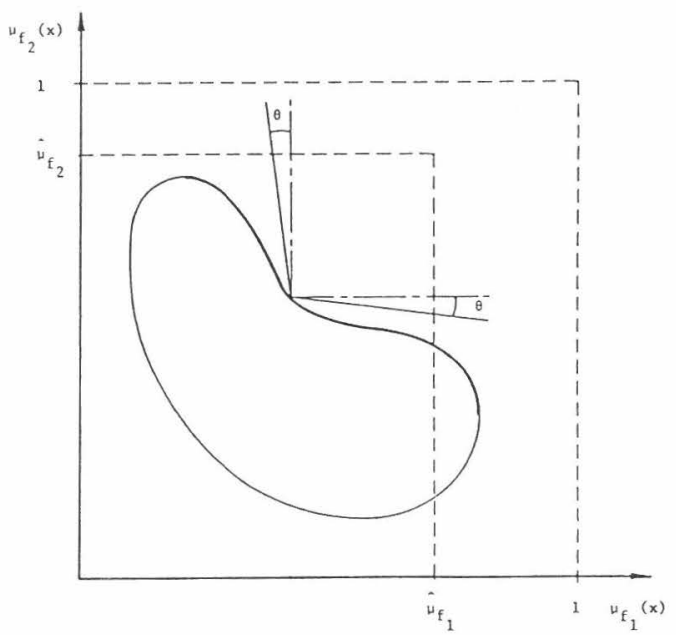

Fig. 1. Isoquants of the minimax problem and the augmented minimax problem.

Naturally, $\rho$ should be a sufficiently small, but computationally significant, positive scalar. However, for practical purposes, a computationally significant lower bound $\rho$ may be

$$
\rho=10^{-(a-b)}
$$

where $a$ is the precision figure of the computer, and $b$ is the figure of each membership values which the DM can distinguish. In most cases, a computationally significant value of $\rho=10^{-3} \sim 10^{-5}$ should suffice.

Now given the local Pareto optimal solution for the reference membership values specified by the DM by solving the corresponding augmented minimax problem (3), the DM must either be satisfied with the current local Pareto optimal solution, or act on this solution by updating his/her reference membership values.

In order to help the DM express his/her degree of preference, trade-off information between a standing membership function $\mu_{f_{1}}(x)$ and each of the other membership functions is very useful. Such a trade-off between $\mu_{f_{1}}(x)$ and $\mu_{f i}(x)$ for each $i=2, \cdots, k$ is easily obtainable since it is closely related to the strict positive Lagrangian multipliers of the augmented minimax problem (3). Let the Lagrangian multipliers associated with the constraints of the augmented minimax problem (3) be denoted by $\lambda_{i}$, $i=1, \cdots, k$. If all $\lambda_{i}>0$ for each $i$, then by extending the results in Haimes and Chankong [12], it can be proved that the following expression holds ${ }^{1}$

$$
\partial \mu_{f_{i}}(x) / \partial \mu_{f_{1}}(x)=-\lambda_{1} / \lambda_{i}, \quad i=2, \cdots, k
$$

\footnotetext{
${ }^{1}$ Mathematically, it is assumed that 1$)\left(v^{*}, x^{*}\right)$ is a regular point of the constraints of the augmented minimax problem 3 ); 2) the second-order sufficiency conditions are satisfied at $\left(v^{*}, x^{*}\right)$; and 3$)$ there are no degenerate constraints at $\left(v^{*}, x^{*}\right)$, where $\left(v^{*}, x^{*}\right)$ is an optimal solution to the augmented minimax problem (3).
}

The formal proof of this relation can be found in Yano and Sakawa [39] and will therefore be omitted. However, geometrically, we can understand it as follows. In $\left(\mu_{f,}, \mu_{f_{2}}, \cdots, \mu_{f}, v\right)$ space, the tangent hyperplane at some point on Pareto surface can be described by

$$
\begin{aligned}
& H\left(\mu_{f_{1}}, \mu_{f_{2}}, \cdots, \mu_{f_{k}}, v\right) \\
& \quad=a_{1} \mu_{f_{1}}+a_{2} \mu_{f_{2}}+\cdots+a_{k} \mu_{f_{k}}+b v=c .
\end{aligned}
$$

The necessary and sufficient condition for the small displacement from this point belongs to this tangent hyperplane is $\Delta H=0$ or equivalently $a_{1} \Delta \mu_{f_{1}}+\mathrm{a}_{2} \Delta \mu_{f_{2}}$ $+\cdots+a_{k} \Delta \mu_{f_{k}}+b \Delta v=0$. For fixed values of $\Delta \mu_{f_{j}}=0$ $(j=2, \cdots, k, j \neq i)$ and $\Delta v=0$ except $\mu_{f_{1}}$ and $\mu_{f_{i}}$, we have $a_{1} \Delta \mu_{f_{1}}+a_{i} \Delta \mu_{f_{i}}=0$. Similarly, for fixed values of $\Delta \mu_{f_{i}}=0(i=1, \cdots, k, i \neq j)$ except $\mu_{f_{j}}$ and $v$, we have $a_{j} \Delta \mu_{f_{j}}+b \Delta v=0$. It follows from the last two relations that

$$
-\frac{\Delta \mu_{f_{i}}}{\Delta \mu_{f_{1}}}=\frac{a_{1}}{a_{i}}=\frac{\left(-a_{1} / b\right)}{\left(-a_{i} / b\right)}=\frac{\left(\Delta v / \Delta \mu_{f_{1}}\right)}{\left(\Delta v / \Delta \mu_{f_{i}}\right)} .
$$

Consequently, it holds that

$$
-\frac{\partial \mu_{f_{i}}}{\partial \mu_{f_{1}}}=\frac{\left(\partial v / \partial \mu_{f_{1}}\right)}{\left(\partial v / \partial \mu_{f_{t}}\right)} .
$$

Now using the Lagrangian multipliers $\lambda_{\mathrm{i}}, i=1, \cdots, k$ associated with all the active constraints of the augmented minimax problem (3), we observe that

$$
\frac{\partial v}{\partial \mu_{f_{i}}}=-\lambda_{i}, \quad i=1, \cdots, k .
$$

Hence we have the result (9) as required.

It should be noted here that in order to obtain the trade-off rate information from (9), all the constraints of the augmented minimax problem (3) must be active. Therefore, if there are inactive constraints, it is necessary to replace $\hat{\mu}_{f_{i}}$ for inactive constraints by $\mu_{f_{i}}\left(x^{*}\right)$ and solve the corresponding augmented minimax problem (3) for obtaining the Lagrangian multipliers.

So far we have considered a minimization problem and consequently assumed that the DM has a fuzzy goal such as " $f_{i}(x)$ should be substantially less than $a_{i}$."

In the fuzzy approaches, we can further deal with a more general case where the DM has two types of fuzzy goals, namely fuzzy goals expressed as " $f_{i}(x)$ should be in the vicinity of $b_{i}$ " (called fuzzy equal) as well as " $f_{i}(x)$ should be substantially less than $a_{i}$ " (called fuzzy min). Such a generalized MONLP problem may now be expressed as:

$$
\left.\begin{array}{ll}
\text { fuzzy min } & f_{i}(x)(i \in I) \\
\text { fuzzy equal } & f_{i}(x)(i \in \bar{I}) \\
\text { subject to } & x \in X
\end{array}\right\}
$$

where $I \cup \bar{I}=1,2, \cdots, k$.

In order to elicit a membership function from the DM for the fuzzy goal " $f_{i}(x)$ should be in the vicinity of $b_{i}$," it is obvious that we can use different functions to the left 


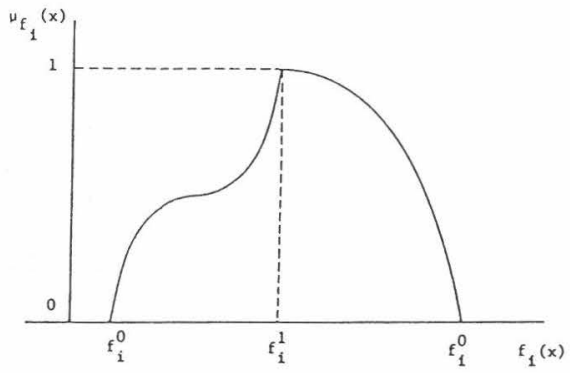

Fig. 2. An example of a fuzzy equal membership function.

and right sides of $b_{i}$. Fig. 2 illustrates the graph of the fuzzy equal membership function where the left function is hyperbolic inverse and the right function is exponential.

Having determined the membership functions for two types of fuzzy goals, if the DM specifies his/her reference membership values, the corresponding augmented minimax problem (3) is solved in order to generate a candidate for the satisficing solution.

When fuzzy equal is included in the fuzzy goals of the $\mathrm{DM}$, it is desirable that $f_{i}(x)$ should be as close to $b_{i}$ as possible. Consequently, the notion of local Pareto optimal solutions defined in terms of objective functions cannot be applied. For this reason, we introduce the concept of local $M$-Pareto optimal solutions which is defined in terms of membership functions instead of objective functions, where $M$ refers to membership.

Definition 3 (local M-Pareto optimal solution): A decision $x^{*} \in X$ is said to be a local $M$-Pareto optimal solution to (11), if and only if there does not exist another $x \in X \cap N\left(x^{*}, r\right)$ such that $\mu_{f_{i}}(x) \geqslant \mu_{f_{i}}\left(x^{*}\right), i=1, \cdots, k$, with strict inequality holding for at least one $i$.

Observe that the set of local Pareto optimal solutions is a subset of the set of local $M$-Pareto optimal solutions. Using the concept of local $M$-Pareto optimality, the following $M$-Pareto version of Theorems 1 and 2 can be obtained.

Theorem 3: The $x^{*} \in X$ is a local $M$-Pareto optimal solution to (11), if and only if there exists $\hat{\mu}_{f}=\left(\hat{\mu}_{f_{1}}, \cdots, \hat{\mu}_{f_{k}}\right)$ such that $x^{*}$ is a local optimal solution to the augmented minimax problem. The proof of this theorem is much like that of Theorems 1 and 2 and thus is omitted.

Following the above discussions, we can now construct the interactive algorithm in order to derive the local satisficing solution for the DM from among the local $M$-Pareto optimal solution set. The steps marked with an asterisk involve interaction with the DM.

Step 0 (Individual Minimum and Maximum): Calculate the individual minimum $f_{i}^{\mathrm{min}}$ and maximum $f_{i}^{\text {max }}$ of each objective function $f_{i}(x)$ under given constraints.

Step $1^{*}$ (Membership Functions): Elicit a membership function $\mu_{f_{i}}(x)$ from the DM for each of the objective functions.

Step 2 (Initialization): Set the initial reference membership values $\hat{\mu}_{f_{i}}^{(1)}=1, i=1, \cdots, k$ and set the iteration index $r=1$.
Step 3 (Local $M$-Pareto Optimal Solution): Set $\hat{\mu}_{f_{i}}=$ $\hat{\mu}_{f_{i}}^{(r)}, i=1, \cdots, k$, solve the corresponding augmented minimax problem to obtain the local $M$-Pareto optimal solution $x^{(r)}, f\left(x^{(r)}\right)$ and the membership function value $\mu_{f}\left(x^{(r)}\right)$ together with the trade-off rate information between the membership functions.

Step 4* (Termination or Updating): If the DM is satisfied with the current levels of $\mu_{f_{i}}\left(x^{(r)}\right), i=1, \cdots, k$ of the local $M$-Pareto optimal solution, exit the program. Then the current local $M$-Pareto optimal solution $f\left(x^{(r)}\right)=\left(f_{1}\left(x^{(r)}\right), \cdots, f_{k}\left(x^{(r)}\right)\right)$ is the local satisficing solution of the DM. Otherwise, ask the DM to update the current reference membership values $\hat{\mu}_{f_{i}}^{(r)}$ to the new reference membership values $\hat{\mu}_{f_{i}}^{(r+1)}, i=1, \cdots, k$ by considering the current values of the membership functions together with the trade-off rates between the membership functions. Set $r=r+1$ and return to Step 3. Here it should be stressed for the DM that any improvement of one membership function can be achieved only at the expense of at least one of the other membership functions.

\section{An Interactive Computer Program}

Fuzzy satisficing decisionmaking processes for multiobjective nonlinear programming (MONLP) problems include eliciting a membership function for each of the objective functions and reference membership values from the DM. Thus, interactive utilization of computer facilities is highly recommended. Based on the method described earlier, we have developed a new interactive computer program. Our new package includes graphical representations by which the DM can figure the shapes of his/her membership functions, and he/she can find incorrect assessments or inconsistent evaluations promptly, revise them immediately, and proceed to the next stage more easily.

Our program is composed of one main program and several subroutines. The main program calls in and runs the subprograms with commands indicated by the user. Here we give a brief explanation of the major commands prepared in our program.

MINMAX Displays the calculated local individual minimum and maximum of each of the objective functions under the given constraints.

MF Elicit a membership function from the DM for each of the objective functions.

GRAPH Depicts graphically the shape of the membership function for each of the objective functions.

GO Derives the satisficing solution for the DM from among the local $M$-Pareto optimal solution set by updating the reference membership values.

STOP Exits the program.

SAVE Saves all the necessary information, which has been put in, in a file.

READ Restores the information which was saved in the file. 
In our computer program, the DM can select his/her membership function in a subjective manner by considering the rate of increase of membership of satisfaction from among the following five types of functions: linear [41], exponential, hyperbolic [19], hyperbolic inverse and piecewise linear [13] functions. Then the parameter values are determined through the interaction with the DM. Here, except for hyperbolic functions, it is assumed that $\mu_{f_{i}}(x)=$ 0 if $f_{i}(x) \geqslant f_{i}^{0}$ and $\mu_{f_{i}}(x)=1$ if $f_{i}(x) \leqslant f_{i}^{1}$, where $f_{i}^{0}$ is an unacceptable level for $f_{i}(x)$ and $f_{i}^{1}$ is a totally desirable level for $f_{i}(x)$.

The linear membership function is given by

$$
\mu_{f_{i}}(x)=\left(f_{i}(x)-f_{i}^{0}\right) /\left(f_{i}^{1}-f_{i}^{0}\right) .
$$

The linear membership functior an be determined by asking the DM to specify the two points $f_{i}^{0}$ and $f_{i}^{1}$ within $f_{i}^{\max }$ and $f_{i}^{\min }$.

The exponential membership function is given by

$$
\mu_{f_{i}}(x)=a_{i}\left(1-\exp \left(-\alpha_{i}\left(f_{i}(x)-f_{i}^{0}\right) /\left(f_{i}^{1}-f_{i}^{0}\right)\right)\right) .
$$

The exponential membership function can be determined by asking the DM to specify the three points $f_{i}^{0}, f_{i}^{0.5}$ and $f_{i}^{1}$ within $f_{i}^{\max }$ and $f_{i}^{\min }$, where $\alpha_{i}$ is a shape parameter, and $f_{i}^{a}$ represents the value of $f_{i}(x)$ such that the degree of membership function $\mu_{f_{i}}(x)$ is $a$.

The hyperbolic membership function is given by

$$
\mu_{f_{i}}(x)=(1 / 2) \tanh \left(\alpha_{i}\left(f_{i}(x)-b_{i}\right)\right)+(1 / 2) .
$$

The hyperbolic membership function can be determined by asking the DM to specify the two points $f_{i}^{0.25}$ and $f_{i}^{0.5}$ within $f_{i}^{\max }$ and $f_{i}^{\min }$, where $\alpha_{i}$ is a shape parameter, and $b_{i}$ is associated with the point of inflection.

The hyperbolic inverse membership function is given by

$$
\mu_{f_{i}}(x)=a_{i} \tanh ^{-1}\left(\alpha_{i}\left(f_{i}(x)-b_{i}\right)\right)+(1 / 2)
$$

The hyperbolic inverse membership function can be determined by asking the DM to specify the three points $f_{i}^{0}$, $f_{i}^{0.5}$ within $f_{i}^{\max }$ and $f_{i}^{\min }$, where $\alpha_{i}$ is a shape parameter, and $b_{i}$ is associated with the point of inflection.

The piecewise linear membership function is given by

$$
\mu_{f_{i}}(x)=\sum_{j=1}^{N_{i}} \alpha_{i j}\left|f_{i}(x)-g_{i j}\right|+\beta_{i} f_{i}(x)+\lambda_{i}
$$

where

$$
\begin{aligned}
\alpha_{i j} & =\left(t_{i, j+1}-t_{i j}\right) / 2 \\
\beta_{i} & =\left(t_{i, N_{i}+1}+t_{i 1}\right) / 2, \\
\lambda_{i} & =\left(s_{i, N_{i}+1}+s_{i 1}\right) / 2 .
\end{aligned}
$$

Here, it is assumed that $\mu_{f_{i}}(x)=t_{i r} f_{i}(x)+s_{i}$, (i.e., $t_{i r}$ is the slope and $s_{i r}$ is the $y$-intercept) for each segment $g_{i r-1} \leqslant f_{i}(x) \leqslant g_{i r}$. The piecewise linear membership function can be determined by asking the DM to specify the degree of membership in each of several values of objective functions within $f_{i}^{\max }$ and $f_{i}^{\min }$.

\section{AN APPLICATION TO ENVIRONMENTAL PROBLEMS}

Consider the application of the proposed method to an industrial area in Japan. The middle part of Osaka Prefecture is one of the most highly industrialized areas in Japan. Osaka City, which is the second largest industrial and commercial area in Japan, contains many small rivers which are branches of the Yodo River. The Yodo River is an important source of drinking water for Osaka's residents, but water pollution in the Yodo River basin has become increasingly serious. Air pollution is also at critical level in the greater Osaka area. In addition, the water supply capacity is limited in this area. Moreover, the limitations of land use in this area are obvious, since it is one of the most populous areas in Japan [23].

Here the industrial pollution control problem for Osaka City is formulated as the following three objective optimization problem [24], [26], [28]:

$$
\left.\begin{array}{ll}
\operatorname{maximize} & f_{1}=\sum_{j=1}^{n} A_{j} K_{j}^{1-b_{j}} L_{j}^{b_{j}} \\
\operatorname{minimize} & f_{2}=\sum_{j=1}^{n}\left(\omega_{1 j} / k_{j}\right) K_{j} \\
\operatorname{minimize} & f_{3}=\sum_{j=1}^{n}\left(\omega_{2 j} / k_{j}\right) K_{j}
\end{array}\right\}
$$

subject to

$$
\begin{aligned}
\sum_{j=1}^{n}\left(\gamma_{i j} / k_{j}\right) K_{j} & \leqslant \Gamma_{i}, \quad i=1,2 \\
q_{2} & \leqslant\left(\sum_{j=1}^{n} K_{j}\right) /\left(\sum_{j=1}^{n} L_{j}\right) \leqslant q_{1} \\
\alpha K_{j 0} & \leqslant K_{j} \leqslant \beta K_{j 0} \\
\alpha^{\prime} L_{j 0} & \leqslant L_{j} \leqslant \beta^{\prime} L_{j 0}
\end{aligned}
$$

where

$j$ an industry $(j=1, \cdots, n ; n=20)$

$K_{j}$ capital value (book value of tangible fixed assets) in industry $j$

$K_{j 0}$ actual capital value in industry $j$

$L_{j}$ number of employees in industry $j$

$L_{j 0}$ actual number of employees in industry $j$

$\omega_{i j}$ unit load of chemical oxygen demand (COD) $(i=1)$ or sulphur dioxide $\left(\mathrm{SO}_{2}\right)(i=2)$ per industrial shipment in industry $j$

$\gamma_{i j}$ resource coefficient for land $(i=1)$ or water $(i=2)$ per industrial shipment in industry $j$

$k_{j}$ capital coefficient, namely capital value per unit of shipments in industry $j$

$\Gamma_{i}$ restriction for land $(i=1)$ or water $(i=2)$

$q_{i}$ upper $(i=1)$ or lower $(i=2)$ bound for the overall capital intensity (ratio of total capital value to total number of employees) 
$b_{j}, A_{j} \quad$ parameters of the production function for each industry $j$, and

$\alpha, \beta, \alpha^{\prime}, \beta^{\prime}$ parameters which represent friction (resistance) in the transfer of capital and labor.

The objective function $f_{1}$ is a Cobb-Douglas type of production function which is homogeneous of degree one and thus if to each factor the value of its marginal product is paid, total output is distributed between capital and labor in the production $1-b_{j}$ and $b_{j}$, respectively. This value should be maximized so as to increase the total production. The objective function $f_{2}$ is the total amount of $\mathrm{COD}$ and should be minimized so as to decrease the water pollution. The objective function $f_{3}$ is the total amount of $\mathrm{SO}_{2}$ and should be minimized so as to decrease the air pollution. Constraints (13) are resource constraints, each of which is a land or water resource constraint. Constraint (14) is the technical constraint, which shows capital intensity as a whole. This has been utilized to indicate the direction of technological changes occurring as a result of the reformation of the industrial structure. Constraints (15) and (16) are frictional constraints: because drastic changes in the industrial structure are not desirable, frictional coefficients are imposed to provide upper and lower bounds for each decision variable. The problem is to find the compromise or satisficing allocation of production factors (capital and labor) to each industry under constraints (13) to (16).

The resource restrictions $\Gamma_{1}$ and $\Gamma_{2}$ in the constraints (13) were assumed to be $\Gamma_{1}=232,200, \Gamma_{2}=200,000$. The parameters $q_{1}$ and $q_{2}$ were supposed to be 1.4 and 0.9 , respectively. The parameter for capital and labor, $\alpha, \alpha^{\prime}$, and $\beta, \beta^{\prime}$, were assumed to be $\alpha=\alpha^{\prime}=0.903, \beta=\beta^{\prime}=$ 1.070. The parameters for $A_{j}, b_{j}, i_{j}$ and $\omega_{i j}, \gamma_{i j}$ are shown in Table I and Table II, respectively. The code numbers of the industrial classification are explained in Table III. The sources for these data have been obtained mainly from Statistical Office of Osaka Prefecture [35], the Ministry of International Trade and Industry [21], and the Osaka Bureau of Trade and Industry [22].

In applying our computer program to this problem, suppose that the interaction with hypothetical DM establishes the following membership function and the corresponding assessment values for the three objective functions

$f_{1}:$ linear, $\left(f_{1}^{0}, f_{1}^{1}\right) \quad=(4800000,5020000)$

$f_{2}$ : hyperbolic, $\left(f_{2}^{0.25}, f_{2}^{0.5}\right)=(147000,145000)$

$f_{3}$ : exponential, $\left(f_{3}^{0}, f_{3}^{0.5}, f_{3}^{1}\right)=(110000,104000,102000)$.

In Fig. 3 the interaction processes using the time-sharing computer program under TSS of an ACOS- 1000 digital computer at the computer center of Kobe University in Japan are explained-especially for the first iteration through the aid of some of the computer outputs. In this interaction, the initial values of the decision variables $x \triangleq$ $\left(K_{1}, \cdots, K_{20}, L_{1}, \cdots, L_{20}\right)$ are set at their lower bounds.

Pareto optimal solutions are obtained by solving the augmented minimax problem using the revised version of
TABLE I

Calculated Values of Parameters $a_{j}, b_{j}$, AND $k_{j}$

\begin{tabular}{crcc}
\hline \hline Industry & \multicolumn{1}{c}{$A_{j}$} & $b_{j}$ & $k_{j}$ \\
\hline 1 & 10.9000 & 0.1145 & 0.1195 \\
2 & 8.6200 & 0.1391 & 0.1160 \\
3 & 15.3900 & 0.1566 & 0.0716 \\
4 & 6.1000 & 0.1779 & 0.1599 \\
5 & 9.9900 & 0.1723 & 0.0926 \\
6 & 5.4600 & 0.1540 & 0.1868 \\
7 & 7.2200 & 0.2291 & 0.1824 \\
8 & 7.9100 & 0.1294 & 0.1400 \\
9 & 6.7300 & 0.1479 & 0.1735 \\
10 & 9.5200 & 0.1737 & 0.1125 \\
11 & 15.2200 & 0.1445 & 0.0670 \\
12 & 6.1300 & 0.1865 & 0.1926 \\
13 & 6.4900 & 0.1216 & 0.1746 \\
14 & 8.1800 & 0.0870 & 0.1077 \\
15 & 6.8500 & 0.1981 & 0.1486 \\
16 & 7.4300 & 0.2000 & 0.1659 \\
17 & 9.6700 & 0.1588 & 0.1020 \\
18 & 7.3600 & 0.1841 & 0.1491 \\
19 & 7.0000 & 0.2107 & 0.1394 \\
20 & 8.4700 & 0.1677 & 0.1228 \\
\hline
\end{tabular}

TABLE II

Calculated Values of Parameters $\omega_{i j}$ AND $\gamma_{i j}$

\begin{tabular}{ccccc}
\hline \hline Industry & $\mathrm{COD}$ & $\mathrm{SO}_{2}$ & Land & Water \\
\hline 1 & 0.07875 & 0.00822 & 0.0244 & 0.0407 \\
2 & 0.03111 & 0.02235 & 0.0718 & 0.1292 \\
3 & 0.03110 & 0.02235 & 0.0219 & 0.0072 \\
4 & 0.00142 & 0.00076 & 0.1024 & 0.0324 \\
5 & 0.00142 & 0.00076 & 0.0244 & 0.0121 \\
6 & 0.21680 & 0.06751 & 0.0487 & 0.1564 \\
7 & 0.07133 & 0.05218 & 0.0105 & 0.0154 \\
8 & 0.07133 & 0.05218 & 0.0429 & 0.0599 \\
9 & 0.03466 & 0.01505 & 0.1461 & 0.0212 \\
10 & 0.02592 & 0.00413 & 0.0553 & 0.0549 \\
11 & 0.02592 & 0.00413 & 0.0468 & 0.0542 \\
12 & 0.00198 & 0.07963 & 0.1087 & 0.0617 \\
13 & 0.00587 & 0.02136 & 0.0773 & 0.0562 \\
14 & 0.00084 & 0.03055 & 0.0354 & 0.0373 \\
15 & 0.00116 & 0.00778 & 0.0589 & 0.0293 \\
16 & 0.00083 & 0.00340 & 0.0464 & 0.0129 \\
17 & 0.00105 & 0.00243 & 0.0235 & 0.0133 \\
18 & 0.00073 & 0.00116 & 0.0702 & 0.0267 \\
19 & 0.00367 & 0.00228 & 0.0451 & 0.0324 \\
20 & 0.00864 & 0.00228 & 0.0354 & 0.0258 \\
\hline
\end{tabular}

TABLE III

CLASSIFICATION OF INDUSTRIES

\begin{tabular}{clcl}
\hline \hline Code & \multicolumn{1}{c}{ Industries } & Code & \multicolumn{1}{c}{ Industries } \\
\hline 1 & Foods & 11 & Leather products \\
2 & Textile mill products & 12 & Clay and stone products \\
3 & Apparel products & 13 & Iron and steel \\
4 & Lumber and products & 14 & Nonferrous metals \\
5 & Furnitures & 15 & Fabricated metal products \\
6 & Pulp and paper products & 16 & Machinery \\
7 & Printing and publishing & 17 & Electrical machinery \\
8 & Chemicals and products & 18 & Transportation equipment \\
9 & Coal and petroleum products & 19 & Precision machinery \\
10 & Rubber products & 20 & Miscellaneous \\
\hline
\end{tabular}


COMMANC:

$=$ Go

INPUT SUFFICIENTLY SMALL POSITIVE SCALAR FOR AUGMENTEO TERM: $=0.001$

INITIATES AN INTERACTION WITH ALL THE INITIAL REFERENCE MEMEERSHIP VALUES ARE

( KUHN-TUCKER CONOITIONS SATISFIED)

PARETO OPTIMAL SOLUTION TÓ THE AUGMENTEO MINIMAX PROELEM FOR INITIAL REFERENCE MEMBERSHIP VALUES

\begin{tabular}{|c|c|c|c|c|c|c|}
\hline & \multicolumn{2}{|c|}{ MEMBERSHIP } & I & \multicolumn{2}{|c|}{ OBJECTIVE } & JE FUNCTION \\
\hline$M\left(F_{1}\right)$ & $=$ & 0.5251 & I & $F(1)$ & $=$ & 4915513. \\
\hline$M\left(F_{2}\right)$ & $=$ & 0.5251 & I & $F(2)$ & $=$ & 144817. \\
\hline$M(F 3)$ & $=$ & 0.5251 & I & $F(3)$ & $=$ & 103885. \\
\hline$x(1)$ & $=$ & 28919. & & $x(2)$ & $=$ & 20749 . \\
\hline$x(3)$ & $=$ & 9132. & & $x(4)$ & $=$ & 14417. \\
\hline$x(5)$ & $=$ & 9178. & & $x(6)$ & $=$ & 33403. \\
\hline$x(7)$ & $=$ & 68254 . & & $x(8)$ & $=$ & 78047. \\
\hline$x(9)$ & $=$ & 1809. & & $x(10)$ & $=$ & 5520. \\
\hline$x(11)$ & $=$ & 4026. & & $x(12)$ & $=$ & 14029 . \\
\hline$x(13)$ & $=$ & 104086 . & & $x(14)$ & $=$ & 25958. \\
\hline$x(15)$ & $=$ & 80583 & & $x(16)$ & $=$ & 87216. \\
\hline$x(17)$ & $=$ & 32812 & & $x(18)$ & $=$ & 38813. \\
\hline$x(19)$ & $=$ & 4896. & & $x(20)$ & $=$ & 28094 . \\
\hline$x(21)$ & $=$ & 25783. & & $x(22)$ & $=$ & 18740. \\
\hline$x(23)$ & $=$ & 19347. & & $x(24)$ & $=$ & 8810. \\
\hline$x(25)$ & $=$ & 8851 . & & $x(26)$ & $=$ & 17157. \\
\hline$x(27)$ & $=$ & 47008 & & $x(28)$ & $=$ & 36539. \\
\hline$x(29)$ & $=$ & 885. & & $x(30)$ & $=$ & 4487. \\
\hline$x(31)$ & $=$ & 5896. & & $x(32)$ & $=$ & 9062. \\
\hline$x(33)$ & $=$ & 30980. & & $x(34)$ & $=$ & 10853. \\
\hline$x(35)$ & $=$ & 56420. & & $x(36)$ & $=$ & 56002. \\
\hline$x(37)$ & $=$ & 28597 & & $x(38)$ & $=$ & 19891. \\
\hline$x(39)$ & $=$ & 4437. & & $x(40)$ & $=$ & 24280. \\
\hline
\end{tabular}

TRAOE-OFFS AMONG MEMBERSHIP FUNCTIONS

$-\operatorname{LM}\left(F_{2}\right) / O M\left(F_{1}\right)=\quad 2.8539$

$-[M(F 3) / O M(F 1)=$

ARE YOU SATISFIED WITH THE CURRENT MEMBERSHIP VALUES OF THE PARETO OPTIMAL SOLUTIOIN?

$=$ NÓ

ITERATION 2

CONSIDER THE CURRENT MEMBERSHIP VALUES OF

THE PARETO OPTIMAL SOLUTION TOGETHER WITH

THE TRADE-OFFS AMONG THE MEMBERSHIP FUNCTIONS.

THEN INPUT YOUR REFERENCE MEMBERSHIP VALUES

FOR EACH OF THE MEMRERSHIP FUNCTIONS:

$=0.50 .6$ ( 55

Fig. 3. Interactive decisionmaking processes

the generalized reduced gradient (GRG) [17] program called GRG2 [18]. In GRG2 there are two optimality tests: 1) satisfy the Kuhn-Tucker optimality conditions, and 2) satisfy the fractional change condition

$$
\mid \text { FM }- \text { OBJTST } \mid<\text { EPSTOP } \times \mid \text { OBJTST } \mid
$$

for NSTOP time consecutive iterations, where FM is the current objective value, and OBJTST is the objective value at the start of the previous one dimensional search. NSTOP has a default value of 3. In Fig. 3, it is shown that one of these conditions is satisfied.

In this example, at the fourth iteration, the satisficing solution of the DM is derived and the values of the objectives and decision variables are shown in Fig. 4. The CPU time required in this interaction process was 8.003 , and the example session takes about ten minutes.

The satisficing allocation of capital and labor to each industry corresponding to the results obtained by interac-
CONSIJER THE CURRENT MEMRERSHIP VALUES OF THE PARETO OPTIMAL SOLUTION TOGETHER WITH THE TRADE-OFFS AMONG THE MEMRERSHIP FUNCTIONS. THEN INPUT YOUR REFERENCE MEMBERSHIP VALUES FOR EACH OF THE MEMRERSHIP FUNCTIONS: $=0.48 \quad 0.62 \quad 0.57$

( KUHN-TUCKER CONOITIONS SATISFIEO) PARETO OPTIMAL SOLUTION TO THE AUGMENTEO MINIMAX PROBLEM FQIR YOUR REFERENCE MEMBERSHIP VALUES

\begin{tabular}{|c|c|c|c|c|c|c|}
\hline \multicolumn{3}{|c|}{ MEMRERSHIP } & I & \multicolumn{3}{|c|}{ OE.JECTIVE FUNCTION } \\
\hline M(F1) & $=$ & 0.4568 & I & $F(1)$ & $=$ & 4900487. \\
\hline$M(F 2)$ & $=$ & 0.5968 & I & $F(2)$ & $=$ & 144286 \\
\hline$M(F 3)$ & $=$ & 0.5468 & I & $F(3)$ & $=$ & 103752 . \\
\hline$x(1)$ & $=$ & 28579 . & & $x(2)$ & $=$ & 20749. \\
\hline$x(3)$ & $=$ & 9132. & & $x(4)$ & $=$ & 14417. \\
\hline$x(5)$ & $=$ & 9178. & & $x(6)$ & $=$ & 33403. \\
\hline$x(7)$ & $=$ & 68254. & & $x(8)$ & $=$ & 78047. \\
\hline$x(9)$ & $=$ & 1809. & & $x(10)$ & $=$ & 4660. \\
\hline$x(11)$ & $=$ & 3776. & & $x(12)$ & $=$ & 14029 . \\
\hline$x(13)$ & $=$ & 103740 & & $x(14)$ & $=$ & 25958 . \\
\hline$x(15)$ & $=$ & 80583. & & $x(16)$ & $=$ & 87216. \\
\hline$x(17)$ & $=$ & 32812 & & $x(18)$ & $=$ & 38813. \\
\hline$x(19)$ & $=$ & 4896. & & $x(20)$ & $=$ & 28094 . \\
\hline$x(21)$ & $=$ & 25783 & & $x(22)$ & $=$ & 18740 . \\
\hline$x(23)$ & $=$ & 19347. & & $x(24)$ & $=$ & 8810. \\
\hline$x(25)$ & $=$ & 8851. & & $x(26)$ & $=$ & 17157. \\
\hline$x(27)$ & $=$ & 47008 . & & $x(28)$ & $=$ & 36539. \\
\hline$x(29)$ & $=$ & 885. & & $x(30)$ & $=$ & 4487. \\
\hline$x(31)$ & $=$ & 5896. & & $x(32)$ & $=$ & 9062. \\
\hline$x(33)$ & $=$ & 30980. & & $x(34)$ & $=$ & 10853. \\
\hline$x(35)$ & $=$ & 56420. & & $x(36)$ & $=$ & 56002. \\
\hline$x(37)$ & $=$ & 28597 & & $x(38)$ & $=$ & 19891. \\
\hline$x(39)$ & $=$ & 4437. & & $x(40)$ & $=$ & 24280 \\
\hline
\end{tabular}

TRADE-OFFS AMONG MEMRERSHIP FUNCTIONS

$\begin{array}{ll}-\left[M(F 2) / \operatorname{LM}\left(F_{1}\right)=\right. & 0.9431 \\ -\operatorname{OM}\left(F_{3}\right) / O M\left(F_{1}\right)= & 1.3559\end{array}$

ARE YOU SATISFIEO WITH THE CURRENT MEMRERSHIP VALUES OF THE PARETO GPTIMAL SOLUTION? $=$ YES

Fig. 4. The satisficing solution of the DM.

tion is summarized in Table IV together with the values in 1975.

The satisficing values for the objective functions can be interpreted as compromised values of the DM between the conflicting objectives which are the maximization of the production function and the minimization of two environmental factors ( $\mathrm{COD}$ and $\mathrm{SO}_{2}$ ). The satisficing solution for the decision variables $K_{j}$ and $L_{j}$ shows the satisficing allocation of capital and labor to each industry. These results show that capital values in industry as a whole are reduced compared with the values in 1975. Capital formation in the coal and petroleum industry and in the chemicals and related products industry is especially severely reduced, and the nonferrous metals industry and the fabricated metal product industry decrease their capital formation. On the other hand, in consumer industries such as the lumber and furniture industries, as well as in machine industries such as the electrical machinery industry, capital formation is promoted.

\section{CONCLUSION}

In this paper, we have proposed an interactive fuzzy satisficing method using the augmented minimax problems in order to deal with the fuzzy goals of the DM in 
TABLE IV

SATisficing Allocation OF CAPITAL and LABOR

\begin{tabular}{crrrr}
\hline \hline & \multicolumn{2}{c}{1975} & \multicolumn{2}{c}{ Proposal } \\
Industry & Capital & Labor & Capital & Labor \\
\hline 1 & 31653 & 22527 & 28579 & 25783 \\
2 & 22981 & 17521 & 20749 & 18740 \\
3 & 10114 & 18088 & 9132 & 19347 \\
4 & 13479 & 8237 & 14417 & 8810 \\
5 & 8581 & 8275 & 9178 & 8851 \\
6 & 36996 & 16041 & 33403 & 17157 \\
7 & 75595 & 43494 & 68254 & 47008 \\
8 & 86440 & 34161 & 78047 & 36539 \\
9 & 2004 & 827 & 1809 & 885 \\
10 & 5161 & 4195 & 4660 & 4487 \\
11 & 3764 & 5512 & 3776 & 5896 \\
12 & 15538 & 8472 & 14029 & 9062 \\
13 & 108036 & 28964 & 103740 & 30980 \\
14 & 28750 & 10147 & 25958 & 10853 \\
15 & 75339 & 52749 & 80583 & 56420 \\
16 & 81541 & 52358 & 87216 & 56002 \\
17 & 30677 & 26736 & 32812 & 28597 \\
18 & 32687 & 18597 & 38813 & 19891 \\
19 & 4577 & 4148 & 4896 & 4437 \\
20 & 26266 & 22701 & 28094 & 24280 \\
\hline
\end{tabular}

multiobjective nonlinear programming problems. In our interactive scheme, after determining the membership functions, the satisficing solution of the DM can be derived by updating his/her reference membership values based on the current values of the membership functions together with the trade-off rates between the membership functions. Furthermore, $M$-Pareto optimality of the generated solution in each iteration is guaranteed. Based on the proposed method, the time-sharing computer program has been written to facilitate the interaction processes.

An application to the industrial pollution control problem in Osaka City demonstrated the feasibility and efficiency of both the proposed method and its interactive computer program by simulating the responses of the hypothetical DM. Although the actual DM for the formulated problem would of course select other values of the three objectives than the ones which were selected by the hypothetical DM used in this paper, the way to iterate and calculate is essentially the same. However, further applications must be carried out in cooperation with a person actually involved in decisionmaking. From such experiences the proposed method and its computer program must be revised. We hope that the proposed method and its extension will become efficient tools for man-machine interactive fuzzy decisionmaking under multiple conflict objectives.

\section{REFERENCES}

[1] V. Chankong and Y. Y. Haimes, Multiobjective Decision Making: Theory and Methodology. New York: North-Holland, 1983.

[2] " "Optimization-based methods for multiobjective decision making: An Overview," Large Scale Systems, vol. 5, no. 1, pp. 1-33, 1983.

[3] A. Charnes and W. W. Cooper, Management Models and Industrial Applications of Linear Programming, vols. I and II. New York: Wiley, 1961

14] "Goal programming and multiple-objective optimizations," European J. Operational Res., vol. 1, no. 1. pp. 39-54. 1977
[5] E. U. Choo and D. R. Atkins, "Proper efficiency in nonconvex multicriteria programming," Math. Oper. Res., vol. 8, no. 3, pp. $467-470,1983$

[6] J. L. Cohon, Multiobjective Programming and Planning. New York: Academic, 1978.

[7] A. M. Geoffrion, "Proper efficiency and the theory of vector maximization," J. Math. Analysis and Appl. vol. 22, no. 3, pp. 618-630, 1968.

[8] A. M. Geoffrion, J. S. Dyer, and A. Feinberg, "An interactive approach for multicriterion optimization, with an application to the operation of an academic department," Management Sci. vol. 19, no. 4, pp. 357-368, 1972.

[9] M. Grauer, A. Lewandowski, and A. P. Wierzibicki, Eds., "Multiobjective and Stochastic Optimization," in Proc. IIASA Task Force Meeting, (IIASA Collaborative Proceedings Series, CP-82S12). Laxenburg, Austria: International Institute for Applied Systems Analysis, 1982

[10] M. Grauer and A. P. Wierzibicki, Eds., Lecture Notes in Economics and Mathematical Systems. Berlin: Springer-Verlag, no. 229, 1984.

[11] Y. Y. Haimes, W. A. Hall, and H. T. Freedman, Multiobjective Optimization in Water Resources Systems: The Surrogate Worth Trade-off Method. New York: Elsevier, 1975.

[12] Y. Y. Haimes and V. Chankong, "Kuhn-Tucker multipliers as trade-offs in multiobjective decision-making analysis," Automatica. vol. 15. pp. 59-72, 1979.

[13] E. L. Hannan. "Linear programming with Multiple Fuzzy Goals," Fuzzy. Sets and Syst, vol. 6, pp. 235-248, 1981

[14] C. L. Hwang and A. S. M. Masud, Multiple Objective Decision Making: Methods and Applications. Berlin: Springer-Verlag, 1979.

[15] J. P. Ignizio, Goal Programming and Extensions. Lexington, MA, Heath. 1976

[16] "Generalized goal programming: An overview," Compt. Ops. Res, vol. 10, no. 4, pp. 277-289, 1983.

[17] L. S. Lasdon, R. L. Fox, and M. W. Ratner, "Nonlinear optimization using the generalized reduced gradient method," Revue Francaise d'Automatique, Informatique et Recherch Operationnelle vol. 3, pp. 73-103, 1974.

[18] L. S. Lasdon, A. D. Waren, and M. W. Ratner, "GRG2 User's Guide," tech. memo., University of Texas, 1980.

[19] H. Leberling "On finding compromise solution in multicriteria problems using the fuzzy min-operator," Fuzzy Sets and Syst. vol. 6 , no. 2, pp. $105-118,1981$

[20] S. M. Lee, Goal Programming for Decision Analysis. Philadelphia: Auerbach, 1972.

[21] 1973 Report on Industrial Land and Water, Research and Statistics Department, Minister's Secretariat, Ministry of International Trade and Industry, Tokyo, Japan (in Japanese), 1973.

[22] Osaka Bureau of Trade and Industry, Input-Output Table for Industrial Pollution Analysis in the Kinki Area in 1973, Osaka Bureau of Trade and Industry, Osaka, Japan (in Japanese), in 1973.

[23] Osaka Big Plan, Osaka Prefecture, Japan (in Japanese), 1973.

[24] M. Sakawa and F. Seo, "Interactive multiobjective decisionmaking for large-scale systems and its application to environmental systems," IEEE Trans. Syst. Man, Cybernetics, vol. SMC-10, pp. 796-806, 1980

[25] M. Sakawa, "An interactive computer program for multiobjective decision making by the sequential proxy optimization technique," Int. J. Man-Machine Studies, vol. 14, pp. 193-213, 1981.

[26] M. Sakawa and F. Seo, "Interactive multiobjective decision making in environmental systems using sequential proxy optimization techniques (SPOT)," Automatica, vol. 18, pp. 155-165, 1982.

[27] M. Sakawa, "Interactive multiobjective decision making by the sequential proxy optimization technique: SPOT," European J. Operational Res., vol. 9, no. 4, pp. 386-396, 1982

[28] M. Sakawa and F. Seo, "Interactive multiobjective decision-making in environmental systems using the fuzzy sequential proxy optimization technique," Large Scale Systems, vol. 4, no. 3, pp. 223-243, 1983.

[29] M. Sakawa and N. Mori, "Interactive Multiobjective decisionmaking for nonconvex problems based on the weighted Tchebycheff norm," Large Scale Systems, vol. 5, no. 1, pp. 69-82, 1983.

[30] M. Sakawa, "Interactive computer programs for fuzzy linear programming with multiple objectives," Int. J. Man-Machine Studies. vol. 18 , no. 5, pp. $489-503,1983$

[31] M. Sakawa and T. Yumine, "Interactive fuzzy decision-making for multiobjective linear fractional programming problems," LargeScale Syst., vol. 5, no. 2, pp. 105-114, 1983 
[32] M. Sakawa, T. Yumine and H. Yano, "An interactive fuzzy satisficing method for multiobjective nonlinear programming problems," (Collaborative Paper CP-84-18), International Institute for Applied Systems Analysis, Laxenburg, Austria, 1984.

[33] M. Sakawa and H. Yano, "An interactive goal attainment method for multiobjective nonconvex problems," in Cybernetics and Systems Research 2, R. Trappl, Ed. Amsterdam: North-Holland, 1984.

[34] M. Sakawa and N. Mori, "Interactive multiobjective decisionmaking for nonconvex problems based on the penalty scalarizing functions,". European J. Operational Res., vol. 17, no. 3, pp. 320-330, 1984.

[35] Industrial Statistics Survey Result Table of Industrial Statistics in 1974, Statistical Office of Osaka Prefecture, Osaka, Japan (in Japanese), 1974.

[36] R. E. Steuer and E. U. Choo, "An interactive weighted Tchebycheff procedure for multiple objective programming," Mathematical Programming, vol. 26, pp. 326-344, 1983.

[37] A. P. Wierzibicki, "The use of reference objectives in multiobjective optimization-theoretical implications and practical experiences," (Working Paper WP-79-66), International Institute for Applied
Systems Analysis, Laxenburg, Austria, 1979.

[38] A. P. Wierzbicki, "A mathematical basis for satisficing decision making," (Working Paper WP-80-90), International Institute for Applied Systems Analysis, Laxenburg, Austria, 1980

[39] H. Yano and M. Sakawa, "Trade-off Rates in the Weighted Tchebycheff Norm Method," Trans. S.I.C.E., vol. 21, no. 3, pp 248-255, 1985 (in Japanese).

[40] M. Zeleny, Multiple Criteria Decision Making. New York: McGraw-Hill, 1982.

[41] H. J. Zimmermann, "Fuzzy programming and linear programming with several objective functions," Fuzzy Sets and Syst., vol. 1 , no. 1 , pp. $45-55,1978$.

[42] - "Fuzzy Mathematical Programming," Compt. Ops. Res. vol. 10 , no. 4, pp. 291-298, 1983.

[43] H. J. Zimmermann, B. R. Gaines, and L. A. Zadeh, Editors, Fuzzy Sets and Decision Analysis, TIMS Studies in the Management Sciences, vol. 12, New York: North-Holland, 1984.

[44] S. Zionts and J. Wallenius, "An interactive programming method for solving the multiple criteria problem," Management $\mathrm{Sci}$, vol. 22, no. 6, pp. 652-663, 1976. 
